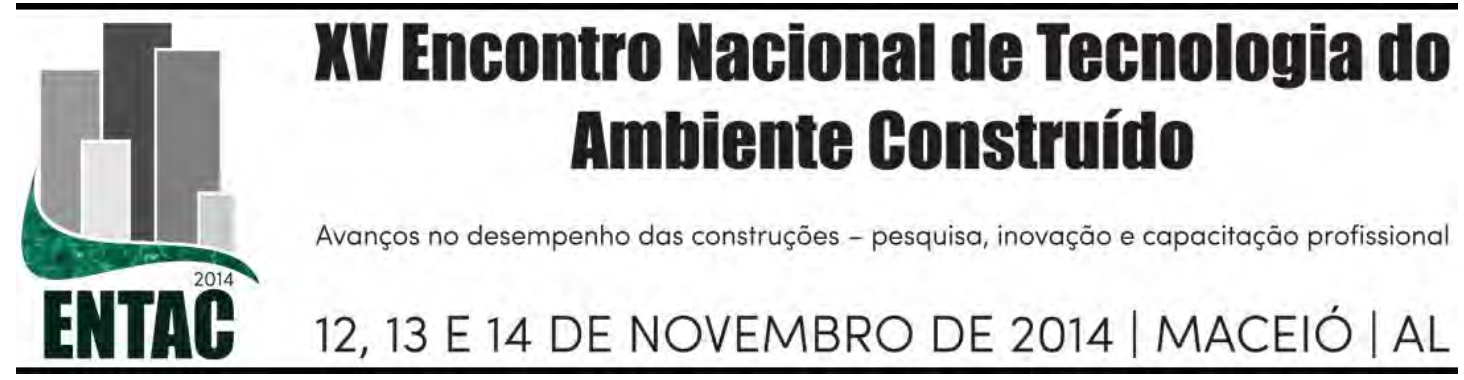

\title{
INFLUÊNCIA DO ENTORNO NAS ILUMINÂNCIAS EM AMBIENTES INTERNOS: estudo de caso no bairro de Manaíra, João Pessoa-PB
}

\author{
LEAL, Lilianne de Queiroz (1); LEDER, Solange Maria (2); \\ SILVA, Luiz Bueno da (3); SOUZA, Erivaldo Lopes de (4) \\ (1) Universidade Federal da Paraíba, \\ e-mail: liliannequeiroz@hotmail.com \\ (2) Universidade Federal da Paraíba, DA/CT/UFPB \\ e-mail: solangeleder@yahoo.com.br \\ (3) Universidade Federal da Paraíba, DEP/CT/UFPB \\ email: silvalb@superig.com.br \\ (4) Técnico de Métodos Quantitativos do DEP/CT/UFPB, \\ email: elopesouza@,hotmail.com
}

\begin{abstract}
RESUMO
O adensamento dos centros urbanos e a verticalização podem afetar expressivamente a disponibilidade da luz natural nas edificações. A componente refletida externa tem papel fundamental sobre os aspectos lumínicos, evidenciando a grande influência do entorno edificado nos cálculos de iluminação natural. Neste contexto, esse estudo propõe analisar a influência do entorno nas iluminâncias em 48 ambientes internos de edifícios residenciais inseridos no bairro de Manaíra em João Pessoa-PB, atribuindo para um mesmo recorte as condições: entorno desobstruído, entorno atual e entorno com a máxima ocupação permitida pela legislação. A análise da pesquisa compreendeu quatro variáveis: 1) Obstrução do entorno; 2) Coeficiente de reflexão das fachadas; 3) Pavimento; e 4) Orientação dos ambientes. O comportamento da luz natural foi simulado por método computacional com uso do software Daysim 3.1e, extraindo dados de iluminância natural

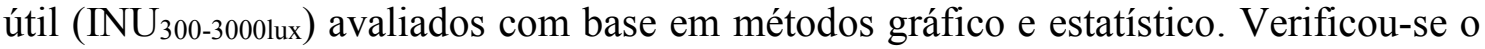
maior percentual de iluminação no edifício isolado e uma diferença estimada de $13 \%$ ao comparar o cenário de máxima ocupação com a situação atual. Os pavimentos mais obstruídos registraram iluminâncias abaixo da faixa útil ( $\mathrm{INU}_{<300 l u x}$ ), tendo percentuais de área iluminada acima de $75 \%$ de INU nas fachadas Noroeste e Nordeste. No caso atual de Manaíra, a futura verticalização do bairro acarretará em reduções de área iluminada, dentro da faixa de iluminância útil, em $21,5 \%$, considerando o cenário com o coeficiente médio de reflexão identificado do bairro, de $58 \%$.
\end{abstract}

Palavras-chave: Iluminação natural, simulação da iluminação, obstrução do céu. 


\begin{abstract}
Densification of urban centres and verticalization can significantly affect the availability of daylight in buildings. The external reflected component has a fundamental role on the luminance aspects, showing the great influence of the built environment in the calculation of daylighting. In this context, this study proposes to analyze the influence of the surroundings in illuminance at 48 indoor environments inserted in residential buildings in Manaíra in city of João Pessoa-PB, assigning conditions to the same land: unobstructed, current and surrounding with maximum occupancy permitted by city's law. Research analysis takes into account four variables: 1) obstruction surrounding; 2) reflectance of surfaces; 3) Floors; and 4) orientation of environments. Daylighting performance was simulated by a computational method with Software Daysim 3.1e, extracting Useful Daylighting Illuminance data (UDI 300 - 3000lux) evaluated by statistical and graphical methods. It was observed a higher percentage of daylighting in the unobstructed building and an estimated difference of $13 \%$ between scenario of maximum occupancy and the current surroundings. The floors more obstructed recorded illuminances below the useful range (UDI <300lux) with percentage of area lit up $75 \%$ of UDI in the Northwest and Northeast facades. In Manairra case, future verticalization will result in reductions of illuminated area, within the range of useful illuminance, at $21.5 \%$, whereas the scenario with the average reflection coefficient identified in Manaíra, of $58 \%$.
\end{abstract}

Keywords: Daylighting, lighting simulation, sky obstruction.

\title{
1 INTRODUÇÃO
}

Existem muitas razões para explorar o uso da luz natural nas edificações, a primeira delas está no seu reconhecimento como fonte abundante e acessível. O seu potencial de aproveitamento varia de acordo com a radiação solar direta, a luz proveniente da abóbada celeste, das reflexões nas obstruções externas e superfícies internas.

Diante das interferências das superfícies externas, a disponibilidade de luz natural pode ser alterada pelo entorno, seja pelas diferenças no gabarito das edificações, na largura das vias, e ainda, na geometria (Tsangrassoulis et al, 1999; Córica e Pattini, 2011). Com efeito, a componente refletida externa depende da geometria urbana, sofrendo modificações em decorrência das propriedades das superfícies e seu coeficiente de reflexão. As particularidades destas superfícies conduzem a frequentes variações na distribuição da luz natural nos espaços internos de edificações circunvizinhas (Li et al, 2010).

Com base nos estudos precedentes sobre o tema, o presente trabalho tem como objetivo avaliar a influência das variáveis de entorno nas iluminâncias em ambientes internos a partir de estudo de caso em edifícios residenciais multipavimentos inseridos em um recorte urbano no bairro de Manaíra na cidade de João Pessoa/PB.

\section{ILUMINAÇÃO NATURAL E ENTORNO}

A necessidade de avaliar a influência do entorno na iluminação dos ambientes internos não é uma preocupação recente. As características do ambiente construído relacionadas ao acesso à fonte de luz natural e ao controle da incidência são definidas pelo tipo e dimensões das aberturas e pelas especificidades do ambiente externo. As variáveis de entorno podem ser consideradas como importante fonte de luz, sobretudo em locais com presença de sol em malha urbana obstruída. Cintra (2011) destaca o adensamento urbano 
e a verticalização como questões que dificultam o aproveitamento da iluminação natural no espaço urbano.

Quando os edifícios são localizados próximos um dos outros, o bloqueio da luz natural pode ser severo, principalmente nos andares inferiores. Li et al (2010) observaram uma redução de $85 \%$ nos níveis de iluminação natural em ambientes obstruídos, para ângulos de obstrução superiores a $60^{\circ}$ relativamente àquela de ângulos inferiores a $10^{\circ}$. Os autores também destacam que a reflexão dos edifícios vizinhos pode ser significativa em ambientes fortemente obstruídos, sobretudo no nível de piso inferior. Ao mesmo tempo em que auxilia no aumento da iluminação no interior dos edifícios, a reflexão ainda pode modificar outras variáveis, quando ultrapassado o limite útil de iluminação, a exemplo do aumento do contraste, ocasionando ofuscamento.

Neste contexto, Córica e Pattini (2011) avaliam a contribuição da fonte natural com base na última reforma realizada no Código de Urbanismo e de Edificações na cidade de Mendoza, Argentina. Através de um estudo de caso se examinou a situação real do cenário, bem como sua configuração de acordo com os indicadores mais atuais para ocupação máxima da área (Figura 1). Em seus resultados preliminares, confirmou-se a contribuição significativa da componente difusa e refletida do entorno urbano de alta densidade. Apesar de tais superfícies promoverem ganho lumínico, os autores alertam ainda sobre as situações em que a exposição ocorre de forma direta, sendo necessário controlar os efeitos de desconforto visual e de sobrecarga térmica, sobretudo em períodos ensolarados.
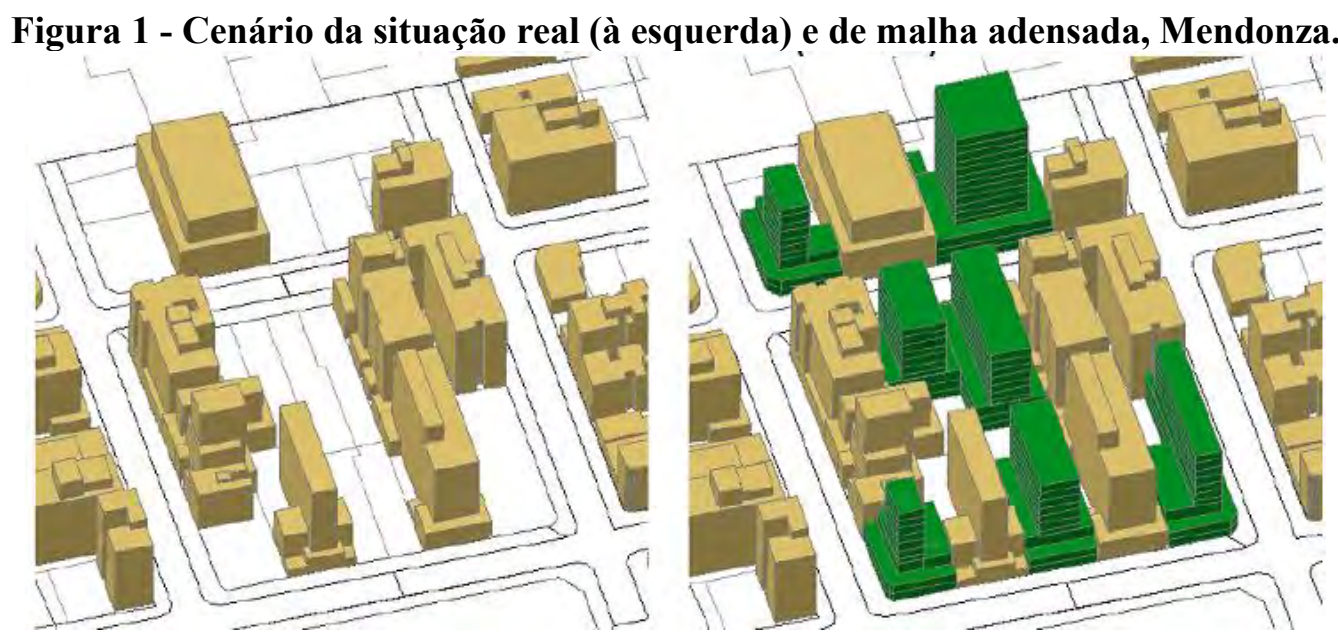

Fonte: Córica e Pattini (2011).

Além do entorno, as características do edifício e do ambiente interno, como pavimento e orientação, são variáveis determinantes para iluminação natural nos espaços construídos. Por sua vez, as aberturas funcionam como componentes de passagem de luz que demandam estudos sistêmicos, em decorrência do grau de exigência no controle dos níveis de conforto desejados (TIBIRICA e FERRAZ, 2005).

\section{MÉTODO}

O procedimento metodológico foi dividido em quatro etapas:

a. Escolha do recorte e caracterização dos cenários;

b. Definição do edifício de análise

c. Simulação computacional;

d. Descrição estatística. 


\subsection{Escolha do recorte e caracterização dos cenários}

O universo da pesquisa foi delimitado por recorte urbano situado no bairro de Manaíra, área litorânea da cidade de João Pessoa - Paraíba (Latitude: $7,11^{\circ}$ Sul e Longitude: $34,86^{\circ}$ Oeste), em função de seu caráter adensado, marcado como uma das localidades mais verticalizadas do município (Figura 2). Inserido no perímetro urbano, o bairro é delimitado pela Zona Adensável Prioritária (ZAP) com o índice de aproveitamento máximo de 4,0 como ocupação do solo nos lotes, segundo definições do Decreto $N^{\circ}$ 5900/ 2007.

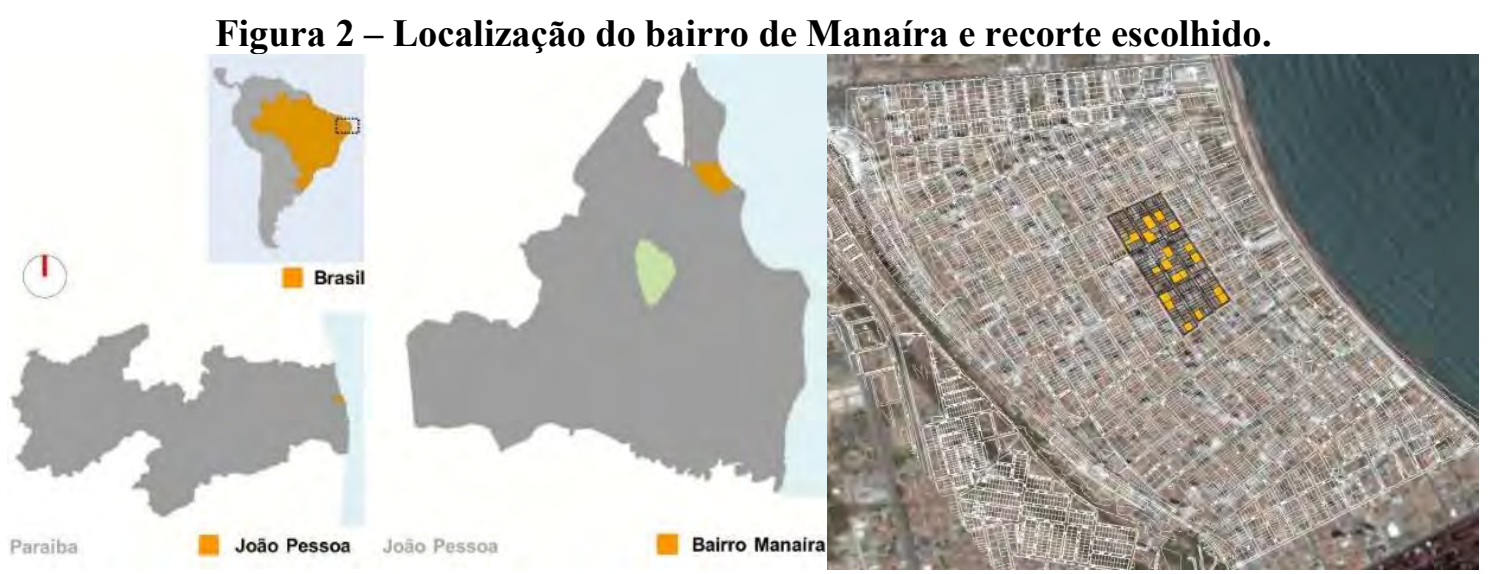

Fonte: Googlemaps adaptado pelos autores.

Os modelos foram caracterizados de acordo com a identificação das variáveis construtivas do bairro (MARTINS, 2013). Dessa forma, foi selecionado um recorte que representa o bairro para servir de base para a modelagem dos cenários. Estes, por sua vez, foram representados em duas categorias: Caso Base - Modelo 1 (Figura 3a), representa a configuração atual do recorte urbano, respeitando o gabarito das edificações e a largura das vias, e os Modelos Hipotéticos, divididos em: Isolado - Modelo 2, no qual a edificação encontra-se sem entorno, e Cenário de ocupação máxima - Modelo 3 (Figura $3 b$ ), que representa o limite máximo de ocupação do solo e do gabarito. A partir destes, foram determinadas as categorias de análise, restritas as características do entorno do Modelo 3, e as variáveis do edifício, comum em todos os modelos. Todos os cenários foram modelados no programa Sketchup v8.0, seguindo as características descritas anteriormente.

Figura 3 - Modelos atual (a) e de máxima ocupação (b), em destaque: edifício em estudo.
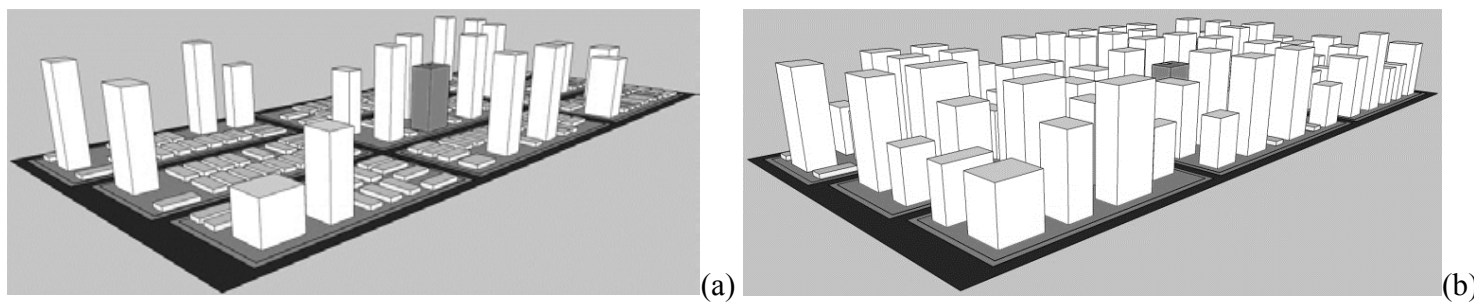

Além do tipo de cenário, também foi adotado como parâmetro a variação da refletividade das superfícies verticais das edificações do entorno. O coeficiente de reflexão médio encontrado no recorte em estudo, de 58\% (LEAL, 2013), foi adotado no cenário atual e máximo, bem como a adoção do coeficiente máximo de $90 \%$ apenas para o cenário máximo, de modo a verificar condições extremas de refletância no entorno. A diferença no coeficiente de reflexão das superfícies externas no cenário de máxima ocupação resultou em dois modelos, denominados de M3A e M3B, para o de refletância média e o de refletância máxima, respectivamente. 


\subsection{Definição do edifício de análise}

A tipologia de análise foi definida por um edifício, cuja representação serviu de parâmetro fixo para todos os modelos de cenário (Figura 4a). Sua configuração correspondeu à predominância no bairro adotando os limites máximos nos percentuais de abertura e $90 \%$ de refletividade para a envoltória. Inserido em quadra e lote centrais, o edifício apresentou dimensões de $39 \mathrm{~m}$ x 30m, resultante de remembramento em três terrenos de $13 \mathrm{mx} 30 \mathrm{~m}$ em quadra e lote centrais, seguindo as orientações mais frequentes no bairro de Manaíra: Sudoeste (SO), Sudeste (SE), Noroeste (NO) e Nordeste (NE).

Figura 4 - (a) Tipologia de análise e (b) planta baixa e os sensores de simulação.
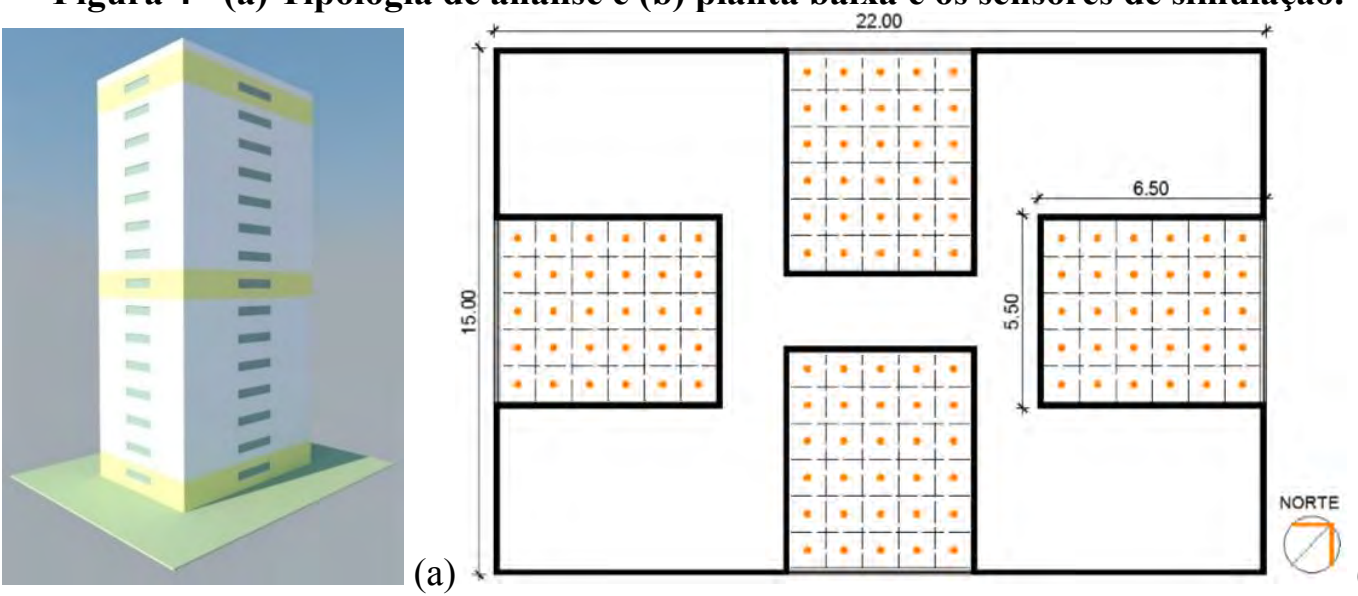

(b)

Considerando apenas três pavimentos para investigação, optou-se pelo andar Térreo, o pavimento Intermediário $\left(8^{\circ}\right)$ e a Cobertura $\left(15^{\circ}\right)$, tendo em cada orientação um ambiente interno situado na porção central da lâmina, esquematizado na Figura 4b. Os quatro ambientes apresentam a geometria retangular com dimensões de 5,50 $\mathrm{m}$ de largura, 6,50 $\mathrm{m}$ de profundidade, pé direito de $2,80 \mathrm{~m}$ de altura e área de $35,75 \mathrm{~m}^{2}$. A parede referente a fachada contém uma abertura que abrange toda a largura do ambiente $(5,50 \mathrm{~m})$, altura de $1,20 \mathrm{~m}$ de altura e peitoril de $0,90 \mathrm{~m}$. Em cada ambiente interno, foi distribuída uma malha de sensores, totalizando 30 pontos para cálculo de iluminância à altura do plano de trabalho de $0,75 \mathrm{~m}$.

\subsection{Simulação computacional}

A verificação da influência do entorno na iluminação dos ambientes internos foi realizada através do Daysim (versão 3.1b), desenvolvido pelo National Research Council Canada (NRCC) e o Fraunhofer Institute for Solar Energy Systems da Alemanha. O programa possibilita analisar o comportamento dinâmico da luz natural em modelos tridimensionais para o período de um ano. Para o processamento das simulações, foram utilizados: arquivo climático anual de 8.760 horas (TRY - Test Reference Year) da cidade de João Pessoa; modelos virtuais dos cenários, dos edifícios e dos ambientes (formato .3ds); malha de sensores de iluminância (formato .pts) e o perfil de ocupação configurado para o horário entre $6 \mathrm{~h}$ e $17 \mathrm{~h}$ (formato .occ).

Os modelos virtuais foram definidos a partir da combinação de quatro variáveis: cenário, refletância das superfícies do entorno, altura do pavimento e orientação. As características de reflexão, comum em todas as simulações, foram configuradas seguindo as recomendações de Lam (1986) e Reinhart (2010). Neste sentido, adotaram-se 18\% no asfalto e $27 \%$ nas calçadas, e para o piso, paredes e teto internos, $30 \%, 70 \%, 80 \%$, respectivamente. As aberturas com $90 \%$ de transmissão luminosa, referente a um vidro de $3 \mathrm{~mm}$ transparente. 


\subsection{Análise estatística}

Os resultados foram analisados através de métodos estatísticos, uma vez que os testes auxiliaram na determinação das variáveis de impacto no percentual de área iluminada, utilizando-se o software R versão 3.0.1. Foram analisados os ambientes com maior percentual de área iluminada de acordo com os valores de iluminância natural útil (INU). Os pontos com INU superior a 75\% do ano (faixa autônoma de 300 a 3000lux) foram quantificados e relacionados com o total de área do ambiente. Adotou-se como referência de ambientes iluminados naturalmente, isto é, os modelos que atingiram percentual de área iluminada superior a 50\%.

Inicialmente foi indicada a distribuição dos dados através de gráficos de caixa (boxplots) por modelo (cenário), aplicando na sequência testes estatísticos - o de normalidade (Shapiro test) e de variância (Chi-quadrado) -, a fim de realizar comparação entre os grupos a partir da medida de variabilidade dos dados. Em complemento, aplicou-se teste de hipóteses, sendo feita a escolha do método em função do resultado da distribuição de probabilidade estatística confirmada com o teste de normalidade. Para tanto, foram escolhidas duas condições: Teste $T$ de Student (paramétrico) ou Wilcoxon Rank (não paramétrico). Com o uso destes testes, pode-se verificar as possíveis chances de semelhanças entre o comportamento das amostras comparadas ou em caso de divergência, suas respectivas estimativas. Quanto a diferença, estimaram-se margens de erro com limites superior e inferior a $5 \%$ do valor esperado por meio dos intervalos de confiança $(95 \%)$.

\section{Resultados}

Com a aplicação do teste estatístico de normalidade e de análise de variância, foram rejeitados os pressupostos quanto à distribuição normal e à variância constante, ambos com valor $\mathrm{p}<2 \times 10^{-6}$. O comportamento das variáveis de cenário quanto ao nível de iluminação dentro da faixa útil (INU>75\%) foi ilustrado na Figura 5.

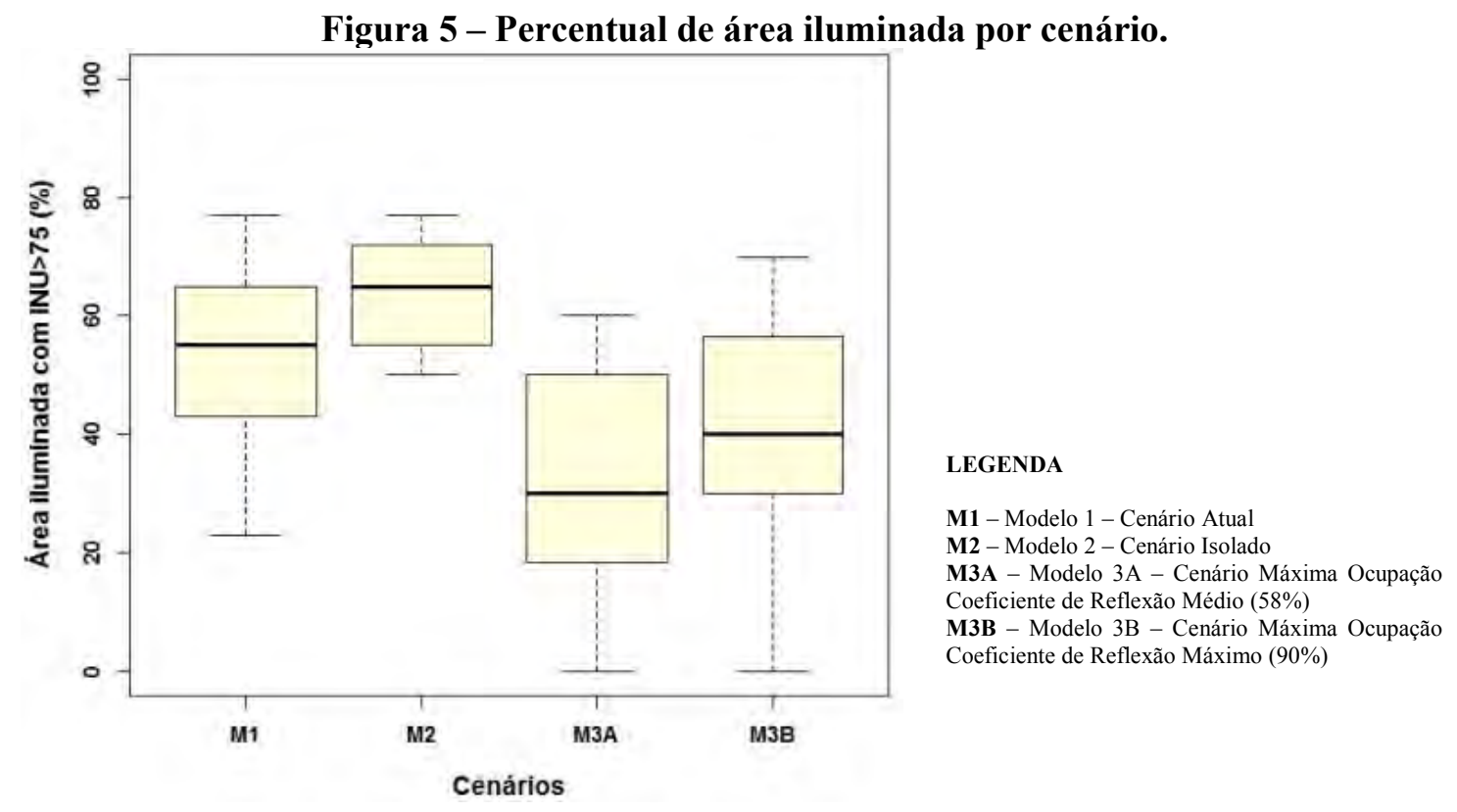

Com a mudança de cenários, considerando todos os pavimentos do edifício, perceberamse alterações nos percentuais de área iluminada dos ambientes internos, sendo mais evidente no Cenário Isolado (M2). Devido à ausência de fatores externos, tais ambientes 
atingiram desempenho lumínico satisfatório com margem entre 50 e $80 \%$ da sua área. Logicamente, os que registraram valores mais baixos sofreram influência das obstruções, sobretudo os Cenários de Máxima Ocupação, cujas fachadas apresentaram coeficiente de reflexão médio (M3A).

Diante da rejeição da normalidade entre os dados, optou-se pelo teste não paramétrico Wilcoxon Rank. Com a aplicação do teste, as comparações múltiplas rejeitaram a hipótese de igualdade dos níveis de iluminação entre os cenários (valor $\mathrm{p}<0,05$ ). Ao comparar o comportamento da iluminação nos ambientes do Cenário Atual (M1) com os do Cenário Isolado (M2), registrou-se um aumento da área iluminada em 10,2\%, confirmando assim a influência do entorno edificado na redução dos níveis de iluminação. Já os ambientes do edifício inserido em cenário de máxima ocupação sofreram redução nos percentuais quando comparados com os do Cenário Atual. Observou-se uma diminuição na área iluminada em $21,5 \%$ e $13 \%$ nos ambientes influenciados pelas fachadas com reflexão média do bairro (M3A) e máxima adotada (M3B), respectivamente (Tabela 01).

Tabela 1 - Comparativo entre percentual de área iluminada referente aos três pavimentos

\begin{tabular}{|c|c|c|}
\hline Cenários & Resultado do teste - Estimativa & \\
\hline Modelo 1 x Modelo 2 & Modelo $1<$ Modelo 2 - Diferença estimada em 10,2\%* & $\mathrm{p}=0,03846$ \\
\hline Modelo 1 x Modelo 3A & Modelo $1>$ Modelo 3A - Diferença estimada em 21,5\%* & $\mathrm{p}=4,3 \times 10^{-5}$ \\
\hline Modelo 1 x Modelo 3B & Modelo $1>$ Modelo 3B - Diferença estimada em 13\%* & $\mathrm{p}=0,001$ \\
\hline Modelo 3A x Modelo 3B & Modelo 3A $<$ Modelo 3B - Diferença estimada em 8,5\%* & $\mathrm{p}=0,0016$ \\
\hline $\begin{array}{l}\text { Modelo } 1 \text { - Cenário Atual } \\
\text { Modelo } 2 \text { - Cenário Isolado }\end{array}$ & \multicolumn{2}{|c|}{$\begin{array}{l}\text { Modelo 3A - Cenário Máxima Ocupação Coeficiente de Reflexão Médio (58\%) } \\
\text { Modelo 3B - Cenário Máxima Ocupação Coeficiente de Reflexão Máximo (90\%) } \\
\text { o nível de significância }(0,05) \text { rejeitando a hipótese de igualdade com estimativa da diferença } \\
\text { de erro de } 5 \% \text {. }\end{array}$} \\
\hline
\end{tabular}

Quando comparados apenas os modelos de máxima ocupação M3A e M3B, o entorno com coeficiente máximo de reflexão (B) contribuiu para um aumento de área iluminada dos ambientes em $8,5 \%$. Neste sentido, observou-se que tanto o grau de obstrução como o coeficiente de reflexão das fachadas de entorno (componente refletida externa) contribuiu para alterações no comportamento da iluminação dos ambientes internos. No que tange a mudança de pavimento, a Figura 6 ilustra diferenças significativas claramente perceptíveis entre os percentuais de área iluminada dos ambientes localizados no andar Térreo, Intermediário e Cobertura.

Figura 6 - Percentual de área iluminada por pavimento.

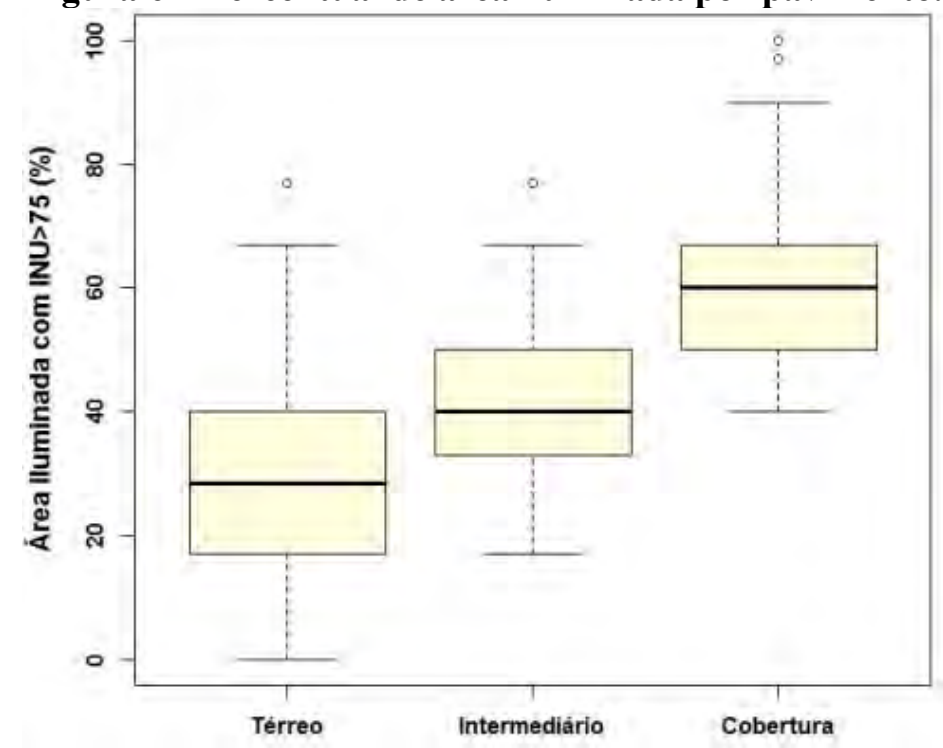


Em função das alturas, registrou-se um aumento proporcional dos níveis de iluminação, sendo os pavimentos inferiores os mais prejudicados, diante das interferências do grau de obstrução. De fato, as diferenças percentuais estimadas entre os pavimentos mais altos (Intermediário e Cobertura) para o Térreo chegaram a uma redução em aproximadamente $13 \%$ e $32 \%$ de área iluminada, respectivamente.

Já a diferença apontada no percentual de área iluminada do Intermediário atingiu redução de 19,3\% quando relacionado com a Cobertura. Os valores obtidos através do teste e da análise dos gráficos foram coerentes e confirmaram estatisticamente o que havia sido constatado empiricamente (Tabela 2).

Tabela 2 - Comparativo entre pavimentos.

\begin{tabular}{|l|l|l|}
\hline Pavimentos & \multicolumn{1}{|c|}{ Resultado do teste - Estimativa } \\
\hline Térreo x Intermediário & Térreo < Intermediário - Diferença em $13,1 \% *$ & $\mathrm{p}=0,00047$ \\
\hline Térreo x Cobertura & Térreo $<$ Cobertura - Diferença em 32\%* & $\mathrm{p}=1,8 \times 10^{-13}$ \\
\hline Intermediário x Cobertura & Intermediário < Cobertura - Diferença em $19,3 \% *$ & $\mathrm{p}=2,263 \times 10^{-8}$ \\
\hline $\begin{array}{l}\text { OBSERVAÇÕES: * Os valores p são menores que o nível de significância }(0,05) \text { rejeitando a hipótese de igualdade com } \\
\text { estimativa da diferença entre as variáveis com margem de erro de } 5 \% .\end{array}$ \\
\hline
\end{tabular}

Além dos três pavimentos, outra condicionante que influiu na alteração dos níveis lumínicos dos espaços internos avaliados pelo percentual de área iluminada foi a orientação (Figura 7). É possível observar que dentro da amostra analisada, os ambientes da fachada Sudoeste (SO) configuraram comportamentos mais atípicos com menor variabilidade e menores percentuais, alcançando valores entre 30 e $50 \%$ de área iluminada em $50 \%$ dos dados. Analisando as demais orientações, verificou-se que os percentuais atingiram faixas entre 30 e $65 \%$, com distribuições similares nas fachadas Noroeste e Nordeste, não apenas pela medida de tendência central - mediana (40\%), mas também pelos valores mínimos e máximos encontrados (20 e 80\%).

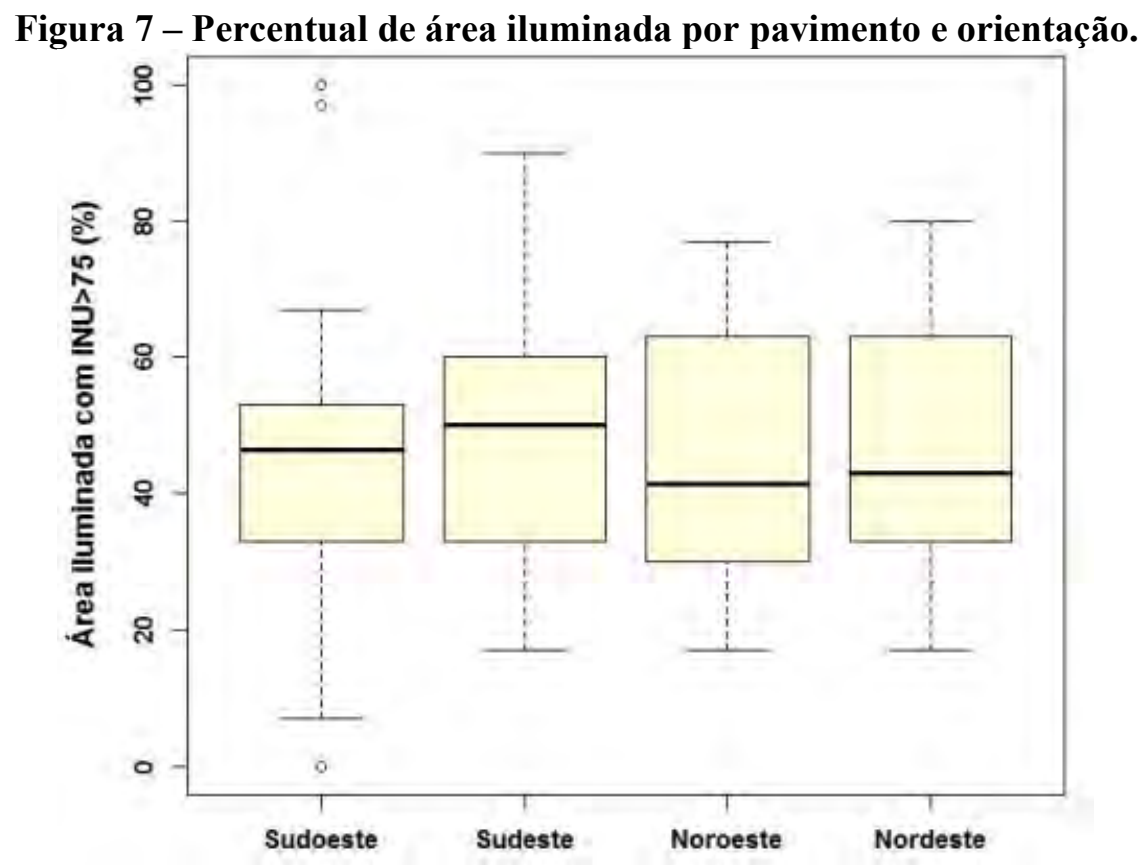

Através do teste de comparação entre as orientações (Wilcoxon Rank), verificou-se que as categorias desta variável, em sua maioria, não possuem diferenças estatisticamente significativas. Diante da margem de erro de $\pm 5 \%$, os intervalos de confiança para os comparativos incluíram o valor zero como uma das estimativas da diferença. Sob esta condição, constatou-se que as orientações Sudoeste, Noroeste e Nordeste apresentaram 
grandes chances de igualdade. No entanto, a fachada Sudoeste e Sudeste rejeitaram tal hipótese, sendo estas as únicas com registro de aproximadamente $6 \%$ de diferença entre as áreas iluminadas (Tabela 3).

Tabela 3 - Comparativo entre pavimentos e orientação.

\begin{tabular}{|l|l|l|}
\hline Orientações & \multicolumn{1}{|c|}{ Resultado do teste - Estimativa } \\
\hline Sudoeste x Sudeste & Sudoeste $<$ Sudeste - Diferença estimada em $6 \%$ & $\mathbf{p}=0,02963$ \\
\hline Sudoeste x Noroeste & Sudoeste $=$ Noroeste* & $\mathbf{p}=\mathbf{0 , 3 2 8}$ \\
\hline Sudoeste x Nordeste & Sudoeste $=$ Nordeste* & $\mathbf{p}=\mathbf{0 , 1 1 5}$ \\
\hline $\begin{array}{l}\text { OBSERVAÇÕES: * Os valores p são maiores que o nível de significância }(0,05) \text { confirmando a hipótese de igualdade, de } \\
\text { acordo com o Teste de Hipóteses Wilcoxon Rank. }\end{array}$
\end{tabular}

\section{CONCLUSÕES}

Esta pesquisa possibilitou comparar o desempenho lumínico de ambientes internos sob diferentes condições de entorno. No caso do recorte urbano em estudo, a implantação das taxas máximas de ocupação permitidas pela atual legislação, procedeu em redução de área iluminada ao comparar com o cenário atual. Consequentemente, ocorreu um aumento em 10,2\% para condições de edifício isolado. Este, por sua vez, apresentou comportamento semelhante aos ambientes localizados no último pavimento em condição máxima de adensamento. O potencial de melhoria com o aumento do coeficiente de reflexão de edifícios vizinhos (Modelo 3A x 3B) se mostrou próximo aos resultados encontrados na situação de entorno atual e desobstruído (Modelo 1 x 2) com influência direta do sol.

Os pavimentos localizados nos andares inferiores apresentaram menores iluminâncias, quando comparados com os pavimentos intermediários e a cobertura, com reduções de $13,1 \%$ e $32 \%$, respectivamente. Em relação à orientação, constatou-se que as orientações Sudoeste, Noroeste e Nordeste apresentaram grandes chances de igualdade. No entanto, as fachadas Sudoeste e Sudeste apresentaram diferença de aproximadamente 6\% ocasionada por interferência do sombreamento do entorno.

Esta pesquisa reforçou a importância de se avaliar as interferências externas sobre o desempenho luminoso de ambiente internos. As consequências da adoção de fachadas com alto coeficiente de reflexão em detrimento do cenário isolado acarretaram no aumento do ofuscamento desconfortável, bem como na redução do acesso ao sol. Destaca-se que as decisões de projeto relacionadas com a iluminação natural devem incluir o entorno, tanto em relação à forma, quanto à refletividade das fachadas.

\section{Agradecimentos}

À Capes pelo auxílio financeiro e ao Laboratório de Métodos Quantitativos Aplicados LabMEQA, DEP/CT/UFPB, pelo auxílio na análise estatística.

\section{REFERÊNCIAS}

CINTRA, M. S. Arquitetura e luz natural: A influência da profundidade de ambientes em edificações residenciais. Dissertação de mestrado, FAU-UNB, 2011.

CÓRICA, I; PATTINI, A. Evaluación del acceso a la iluminación natural em edificios de alta densidad edilicia según los indicadores urbanos del código urbano y edificación de la ciudad 
de Mendoza. Encontro Nacional e V Latino Americano de Conforto no Ambiente Construído ENCAC, Búzios-RJ, 2011.

FIELD, A. Descobrindo a estatística usando o SPSS. Porto Alegre: Armed. $2^{\circ}$ Ed, 2009. 112 $113 \mathrm{p}$.

KVAM, P. H.; VIDAKOVIC, B. Nonparametric Statistics with applications to Science and Engineering. Editora Wiley \& Sons, New Jersey, 2007. 115-139 p.

LAM, W. M. C. Sunlighting as formgiver for architecture. Nova Iorque: Van Nostrand Reinhold, 1986.

LEAL, L. Q. Influência das variáveis de entorno e do edifício sobre iluminâncias e ofuscamento nos ambientes internos: um estudo de caso em edifícios residenciais multifamiliares em recorte urbano na cidade de João Pessoa-PB. Dissertação (Mestrado em Arquitetura e Urbanismo) - Programa de Pós-Graduação em Arquitetura e Urbanismo, UFPB, 2013.

LI, D. H. W.; CHEUNG, G. H. W., CHEUNG, K. L.; LAM, T. N. T. Determination of vertical daylight illuminance under non-overcast sky conditions. Building and Environment. V.45, p. 498-508, 2010.

REINHART, C. Tutorial on the use of Daysim simulations for sustainable design. Tutorial Cambridge, Havard University, 2010.

TIBIRICA, A. C. G.; FERRAZ, R. Considerações para projeto de janelas em edificações: Fatores ambientais, normativos e tecnológicos. In: VIII Encontro Nacional e V Encontro Latino-Americano de Conforto no Ambiente Construído. Maceió, AL: ENCAC, 2005. 